Aims To review whether ultrasounds are being performed for Hospice at Home $(\mathrm{H} @ \mathrm{H})$ patients and what impact this has. Method A retrospective service evaluation of the use of the ultrasound in $\mathrm{H} @ \mathrm{H}$ patients over a one-year period.

Results 10 ultrasounds were performed in $\mathrm{H} @ \mathrm{H}$ patients over one year, seven were in the patient's home and three were in hospice outpatient appointments. Nine ultrasounds were performed to assess for ascites and one to assess for urinary retention.

Of the nine ultrasounds performed to assess for ascites, three demonstrated large volume of ascites that was amenable to drainage. Of these three patients, one had a drain inserted on the hospice inpatient unit and two were referred to hospital for drainage. The other six patients were found to have small volume or no ascites. The patient who had an ultrasound to assess for urinary retention was found not to have a distended bladder.

Conclusion The use of ultrasound in $\mathrm{H} @ \mathrm{H}$ patients does influence patient care and supports clinical decision making. The value of hospices performing ultrasounds comes from the ability to avoid unnecessary visits to hospital for ultrasounds, as well as avoiding unnecessary admissions to the hospice inpatient unit or hospital for assessment or drainage of ascites. Rapidly establishing whether a patient has ascites and whether it is amenable to drainage in the community, reduces delays and distress for the patient, as well as reducing the burden on the wider health service.

\section{P-169 WEIGHING PATIENTS IN A HOSPICE SETTING}

${ }^{1} J a n$ Codling, 'Jennie Pickard, 'Alison Phippen, 'Samantha Kay, ${ }^{2}$ Niall Byrne, ${ }^{1}$ Becki Singh, ${ }^{1}$ David Waterman. 'St Ann's Hospice, Heald Green, UK; ${ }^{2}$ Manchester University, Manchester, UK

\subsection{6/bmjspcare-2017-hospice.194}

Background Standard practice at our hospice did not encourage routine weighing of patients on admission, which potentially limited ability to meet best practice standards for medicine management and nutritional assessment.

Aim This project measured if patients were weighed at or soon after admission. Many were prescribed medication where dose was dependent on weight. The opinions of staff and patients towards routine weighing was also investigated.

Methods An audit of 40 patients measured if patients were weighed on admission or a reason for not doing so recorded and whether weight dependent doses were in line with the British National Formulary or other specialist advice.

A staff questionnaire gained the opinions of 79 clinical staff towards weighing patients, their understanding of the reason for weighing, and rationale for their opinions. A patient questionnaire gained the opinions of 38 patients on being weighed and their understanding of the reason for being weighed.

Results 97\% patients did not find being weighed distressing. However, 51\% staff members were opposed to routine weighing. $13 \%$ of patients had a weight recorded. $13 \%$ were prescribed low molecular weight heparin, $80 \%$ of these patients were weighed and only $60 \%$ were on the correct dose.

Conclusions Routine weighing has been introduced for all patients or a recorded reason for not doing so. Clinical staff now receive training that demonstrates the inaccuracy of estimating body weight. An alert sticker is now attached to the medicine chart, for patients prescribed weight dependant medication and a prompt on the shelves where the medication is stored acts as a reminder to check body weight.

There is a plan for regular audits of the weighing of patients to maintain the profile of the importance of weighing and these results will be fed back real time to the clinical teams.

\section{P-170 DETECTING SUICIDAL THOUGHTS IN PALLIATIVE CARE: WHEN DO PATIENTS VOICE THESE THOUGHTS?}

${ }^{1}$ Chloe Choy, ${ }^{2}$ Kathy Burn, ${ }^{2}$ Emma McLachlan, ${ }^{2}$ Emma Hall. ${ }^{1}$ King's College London, London, UK; ${ }^{2}$ St Christopher's Hospice, London, UK

\subsection{6/bmjspcare-2017-hospice. 195}

Background Early identification of suicidal ideation would allow more opportunities for intervention and may ultimately reduce risk of suicide. Knowing when patients are likely to voice suicidal thoughts is important, as it may increase confidence of healthcare professionals to have earlier conversations with their patients regarding suicide risk.

Aim To establish the time period between first assessment and recorded suicidal ideation in a patient population.

Methods 385 patients under the care of inpatient and community palliative care teams at St Christopher's Hospice, Sydenham, were identified to have keyword 'suicide' in their electronic patient records (EPR) during the period of April 2015 to March 2016. In these individuals, EPR was reviewed against inclusion criteria and 124 patients were identified to have documented suicidal ideation. Time from first assessment to detection of suicidal thoughts was calculated.

Results 61\% of patients with expressed suicidal thoughts $(n=76)$ were male, and mean age of those who voiced suicidal thoughts was $67.4 \pm 13.6$ years (mean \pm SD). $69 \%$ $(n=86)$ had a diagnosis of malignancy, and 62\% $(n=77)$ had a previous psychiatric history. $15 \%(n=19)$ of all patients we identified with suicidal thoughts voiced their suicidal ideation at their first assessment. A further 10\% $(n=12)$ voiced these thoughts within the first week of contact, and in $45 \% \quad(n=55)$ suicidal thoughts had been detected within the first month.

Conclusions A large proportion of patients expressed their suicidal ideation early in their contact with the hospice-centred palliative care team. As previously established, asking patients about thoughts of suicide does not increase risk of suicide or self-harm (Gould et al., 2005; Eynan et al., 2014). This data provides further encouragement to explore suicidal thoughts in palliative care patients from first contact.

\section{P-171 COMFORT ROUNDS: TASK-ORIENTATED NURSING OR EFFECTIVE CARE?}

Jo Carby, Rose Davis, Rebekah Ashley, Debbie Bewley, Nathalie Ward, Yvonne Tague. Wigan and Leigh Hospice, Greater Manchester, UK

\subsection{6/bmjspcare-2017-hospice. 196}

Background Active nursing rounds, also known as intentional, care or comfort rounds were first developed in the USA (Struder Group, 2007) and later introduced into UK hospitals in 2012 alongside other measures to improve the quality of nursing care (www.harmfreecare.org). They have been associated with reductions in pressure ulcers, falls and increased 